\title{
A SHORT ACCOUNT OF A YEAR'S WORK AT ONE OF THE JAW INJURIES CENTERS OF THE LONDON COMMAND. ${ }^{1}$
}

\author{
By G. Northeroft.
}

$\mathrm{T}^{\mathrm{H}}$ HIS paper is a review of the work done at one center with a staff working a certain number of hours, and is intended as a guide for future emergencies. Altho the proportion of jaw injuries may be small compared with the number of other injuries received, it has been proved undoubtedly, both here and abroad, that their total number makes the provision of jaw centers in every Command an essential part of an efficiently organized medical system, and is a point that must never again be neglected. A Jaw Injuries Department of a General Hospital seems the best arrangement for the efficient treatment of such wounded. Experience proves that a large number of men suffer from multiple wounds, and have to be closely watched alike by physicians, surgeons, and dental surgeons. Even in special hospitals it has been frequently shown that the hearty cooperation of all three is essential, and, by following the ideal method, unnecessary multiplication of staffs, with the attendant expense, is largely avoided.

It must not be forgotten, however, that the presence of jaw cases in a General Hospital very seriously handicaps those C.O.'s who are anxious to create records for rapidity of cures and the handling of a maximum number of patients. Supposing that the average detention of a man in hospital is three or four weeks, even the simplest fractured jaw tends to

\footnotetext{
${ }^{1}$ Owing to the cost of production, all illustrations of splints and several photographs shown in the epidiascope have been omitted.
}

double this average. It is suggested, therefore, that to avoid the opposition that has sometimes arisen to the reception of jaw injuries, owing to the tediousness of the treatment and the extra strain on the staff, all jaw statistics should be kept separately, so that the otherwise good record of a hospital should not be interfered with.

The appointment of a Dental Officer to such Medical Boards as sit on jaw cases commends itself as a commonsense arrangement, that would simplify the onerous work of the boards, and also the problem of the classification of the wounded. It has been stated that only medically qualified dental surgeons are fitted to undertake the treatment of these cases, but the fact that many of our confreres are doing good work by using their medical skill, and are not being employed in their specialty, shows that, at any rate while the shortage of medical men continues, those dental surgeons without medical qualifications who have the necessary skill are being wisely employed by the authorities where it is known that complimentary medical and surgical cooperation is available. The operations necessary in the treatment of jaw injuries are of a complicated nature, but while splints for other fractures can be supplied ready made, our work demands a specially made apparatus for each individual, however much an operator may confine himself to one method of treatment.

The point I wish to make is that the 
dental laboratory and expert mechanical aid is an additional but an essential part of a Jaw Injuries Department. It is hardly realized, outside our own profession, how faithful and exact must be the work of the laboratory, if endless delays, and the extra suffering entailed, are to be avoided, and therefore, how necessary it is to have good mechanics and plenty of them. Four to every hundred patients is really the minimum that ought to be entertained.

The extreme importance of very highly skilled radiographers for this work cannot be too strongly emphasized. Many unreadable plates are produced, and an enormous amount of time and money wasted thereby; for instance, the production of thin negatives or lateral views taken with the sides of the mandible superimposed, ought seldom, if ever, to occur. But it is none the less true that no attempt at treatment should be made without the use of this great aid, and stereoscopic plates seem preferable to any others. Photographic records where any plastic work is involved are well worth the time and trouble, and should be made a routine for all Jaw Injury Departments. It is astonishing how quickly one forgets the original appearance of a wound after continuously seeing a patient for two or three months progressively improving. Plaster casts have also proved their value in showing the exact contours and assisting the surgeons in carefully planning the amount of tissue necessary to restore lost features as fully as possible.

The cases received at the First London General Hospital Jaw Injuries Department since it was inaugurated at the end of July, 1916, to September 7, 1917, have amounted to 248 , apart from cases seen in consultation on account of dental disease, or others that have been immediately transferred without treatment. The Department was started with two dental surgeons working every day for the first month and with no laboratory, the necessary splint work being produced by one unattached mechanic, and it is a great pleasure to record that the patriotic labor of this one man, working early and late, without hope of fee or reward, did much towards solving many difficulties created by the delay in building our laboratory, which was not completed until January 1, 1917. About that time it became evident that assistance must be obtained for the purely dental work, which is so essential an accompaniment of efficient treatment, and an Army Dental Lieutenant was appointed to attend three half-days a week. An assistant mechanic was next added, and in July, 1917, owing to a great increase in the number of patients, our President was appointed the third consultant. In future it should be laid down as a stringent rule when creating a Department that adequate provision should be made at the start for a contemplated maximum number of patients according to the general arrangements made at any particular hospital. This maximum number should seldom, if ever, be exceeded.

Our statistics cannot, of course, be compared with the number of cases seen at some other centers, but are large enough to demonstrate certain conclusions. Lindemann reports from Düsseldorf that the cases there showed the ratio of fractured mandible to maxilla and mandible and maxilla to be $5: 1: 1$. Our ratio is seen to be practically the same for the mandible to maxilla, but the ratio of mandible to mandible and maxilla is $8 \mathrm{~T} / 2: 1$. This difference is possibly due to the smaller number of cases examined. Of 248 men seen on September $7,1917,{ }^{2} 20$ have been transferred, and 2 have died a few days after admission, with 116 still under treatment; 136 sustained fractures of the mandible only, twenty-five of the maxilla only, and sixteen of both mandible and maxilla. Thirty-four have been

\footnotetext{
'Since that date fifty-eight new cases have been seen and forty-five cases have been discharged, making a total of 306 , and the number still under treatment 129 .
} 
treated for fractured teeth and alveolar process. Seven suffered from closure only, probably due to cicatricial contraction following an injury not treated at this center. Nine nasal cases have been treated in cooperation with a rhinologist. One remarkable dislocation will receive detailed attention later.

Of the 110 men discharged failure to procure bony union has only occurred in twelve cases (almost exactly 10 per cent.), in one of which was a maxilla completely torn from its bed by a piece of shell which was subsequently removed from the right antrum, and measured $1 \frac{1}{2}$ in. long by $1 / 2$ in. sq. When this patient was discharged he could chew satisfactorily but his teeth still felt "velvety" on mastication. Seeing this man had a remarkably good set of teeth for an English soldier it was decided not to render him edentulous in an endeavor to produce a result which after all was problematical. It is curious to note that of these partial failures seven occurred on the left side and involved the angle and part of the ascending ramus, leaving the posterior fragment edentulous, being combined in some cases with paralysis of the injured side, so that mastication on that side was rendered impossible and the insertion of a bone graft of very doubtful utility. One case had lost the anterior third of the tongue, which rendered the management of any bolus of food impossible. Of this series, the most disappointing was a case that showed apparent union according to the X-ray report and broke down on attempting full diet. The jaws were again immobilized, but the patient being transferred at his own request for family reasons, the case was lost sight of. Five of these cases were supplied with modified Villain's bielles, which were working admirably at the time of discharge, giving the patients as full use of the jaws as could reasonably be expected, the ideal aimed at being the management of full diet.
The case of chronic dislocation, being somewhat unique, deserves description. A private, of indifferent physique, was sent to this center on account of repeated bilateral dislocation which became more and more frequent, commencing first in January, 1915. He was first seen in the department on September 1, 1916, and it was said that his jaw had been displaced a very large number of times. As will be readily seen from the models, the lack of dental inspection by an experienced dentist, and a too rigid economy, kept this man as a positive expense to the State for two years, and a source of irritation to the medical staff. He had been supplied with a denture for the maxilla only and the third molars were the only teeth left for natural occlusion. The maxillary molars occluded buccally to the mandibular, so that every time the mastication of food was attempted, the temporo-mandibular ligaments were stretched to such an extent that finally the mandible fell out of its normal position on the slightest provocation. On September 4 the jaws were immobilized to give the stretched ligaments rest. Grave difficulty was experienced with the patient, who seemed very restless under this treatment and was seriously thought at one time to be malingering Fixation by ligatures proved a failure, but the principle was maintained by using upper and lower cap splints on the front teeth. These were being perpetually wrenched off and the jaws again dislocated as late as October 7. On October 10 septic roots were removed from the mandible and new splints constructed covering the whole of the remaining upper and lower teeth. The jaws were then satisfactorily immobilized for four weeks. A lower vulcanite splint was next constructed to aid in mastication and to prevent the too free movement of the jaws until such time had elapsed that dentures could be made. Upper and lower dentures were inserted in March, 1917 , no further dislocation having been 


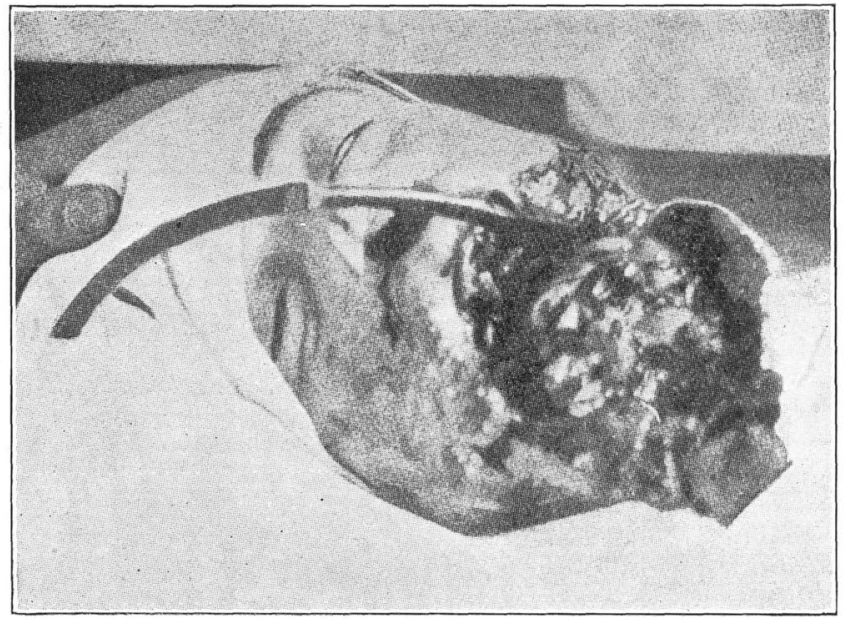

Figure 1.

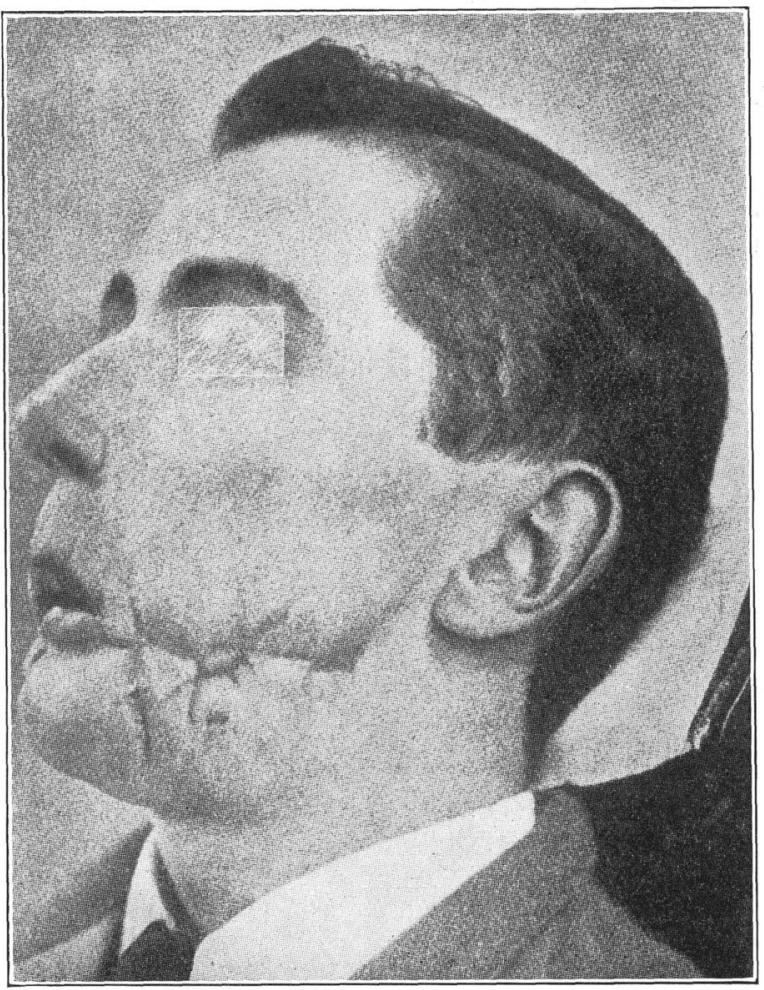

Figure $1 \mathrm{~A}$. 
reported. This case shows how economical and essential it is to have a properly organized system of dental inspection, and illustrates the fact that dentures cannot be turned out like, one might say, boots from a factory.

The cases of fractured teeth and alveolus need no comment except that one has remarked in some of the cases how

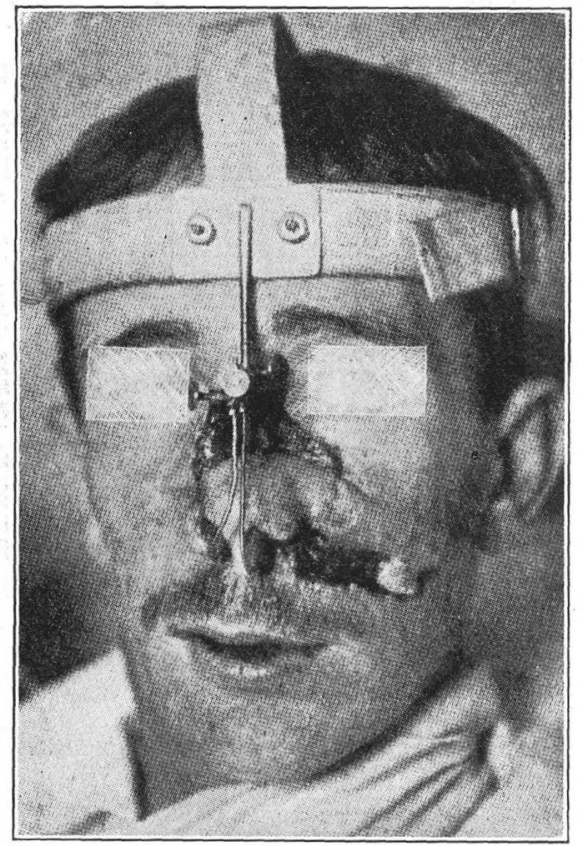

Figure 2.

singularly slow the sockets are to heal, this seeming to show the existence of deep-seated sepsis, or the failure of quick reaction due to excessive trauma or temporary lack of function of the jaws.

Owing to the pleasant cooperation which exists between the dental and surgical staffs it has been our privilege to assist in several nasal cases by constructing the necessary splints and appliances. The cases have not presented any unusual features; the use of vulcanite tapered tubes has been almost exclusively adopted as nose olives which, hav- ing been inserted, were at first attached by their anterior ends to rings of aluminum covered with webbing buckled round the head (Figure 2). Later it was found quite sufficient to hold the olives in by strapping, slipped under a wire loop attached to the anterior end of the vulcanite. One modification may be shown by which the columella was saved after

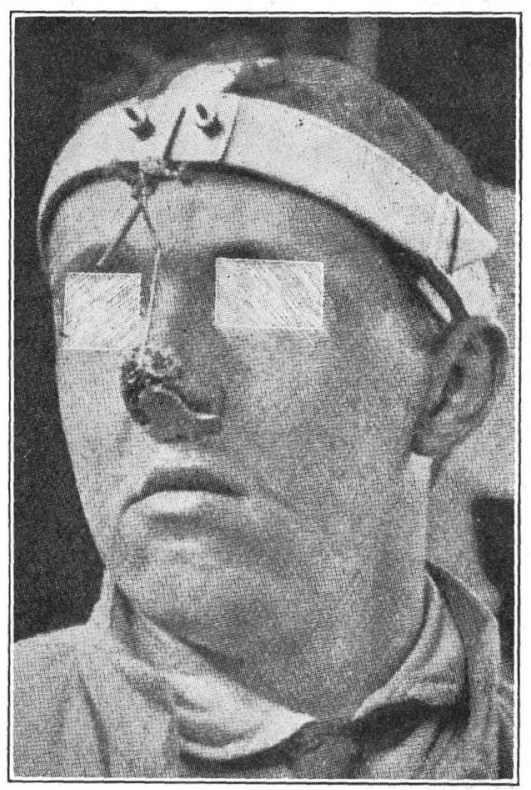

Figure 3

it had become detached and was being bandaged down to the upper lip by the dressing. Olives were first inserted and a support arranged between them. When cicatrization had progressed sufficiently to ensure the nostrils remaining patent, this arrangement was changed for a vulcanite pad attached to an aluminum crown which was used with great success (Figure 3).

Those cases of closure that have not been prevented by using interdental extension pieces on the splints in the first place, have been treated by slowly and 
continuously pressing the jaws open, rather than by forcibly wrenching them, less chance of recurrence being involved.

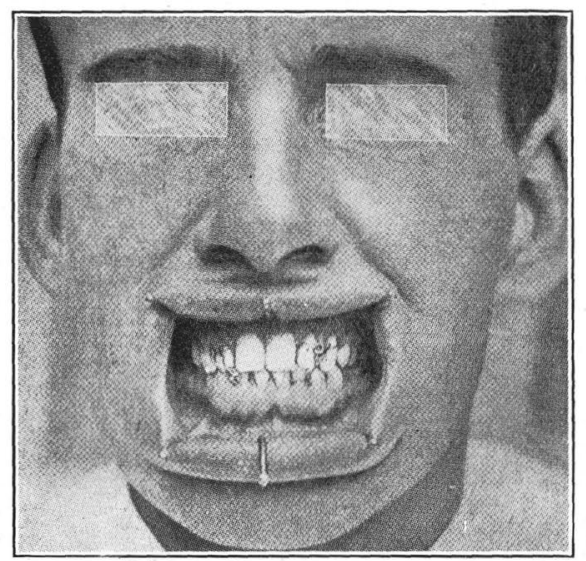

Figure 4.

Seven cases have been treated for this condition only, and in support of the principle laid down, it has been the

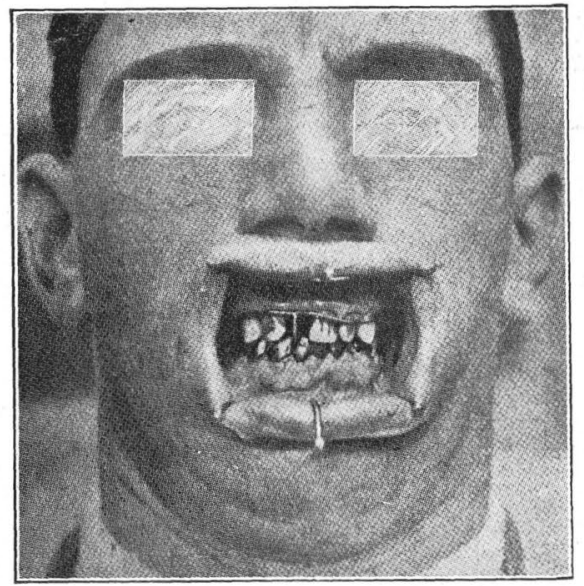

Figure 5 .

custom to construct metal caps for the upper and lower teeth and insert a Talbot coil of heavy piano wire in vertical tubes or vertical upper and hori- zontal lower tubes with stopped ends. The springs should be carefully adjusted to articulated models and care taken that the tubes are equidistant on both sides. This treatment should be supplemented in obstinate cases by the use of screw mouth-openers for ten minutes twice a day.

In the treatment of fractures it has

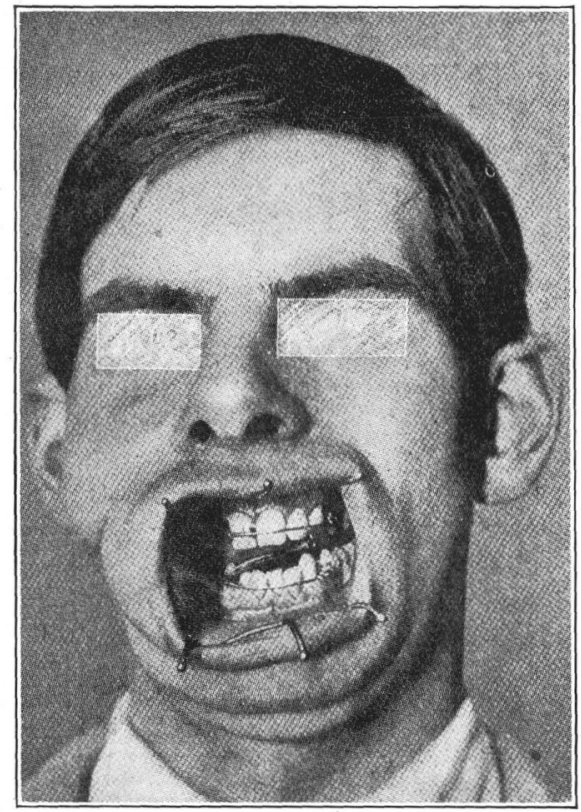

Figure 6.

been our ideal to use whatever method seemed best suited for the case in hand, to free our mind of prejudice, and be catholic in our tastes (Figures 4, 5 and 6).

The first great principle governing the treatment of jaw injuries of whatever type is oral hygiene. To attain this all septic teeth, roots, and other foci of infection should be removed as early as possible. This may sometimes prove a very difficult ideal to attain. For example the removal of a root from a fragment itself so loose that one feels the part is a great- 
er problem than the whole. There are exceptions to every rule, but time and again one has proved the efficiency of this treatment in the earlier response of the tissues to repair. One also recognizes that in extreme cases, when roots or crowns of teeth have been blown into the tongue or cheek, or a foreign body

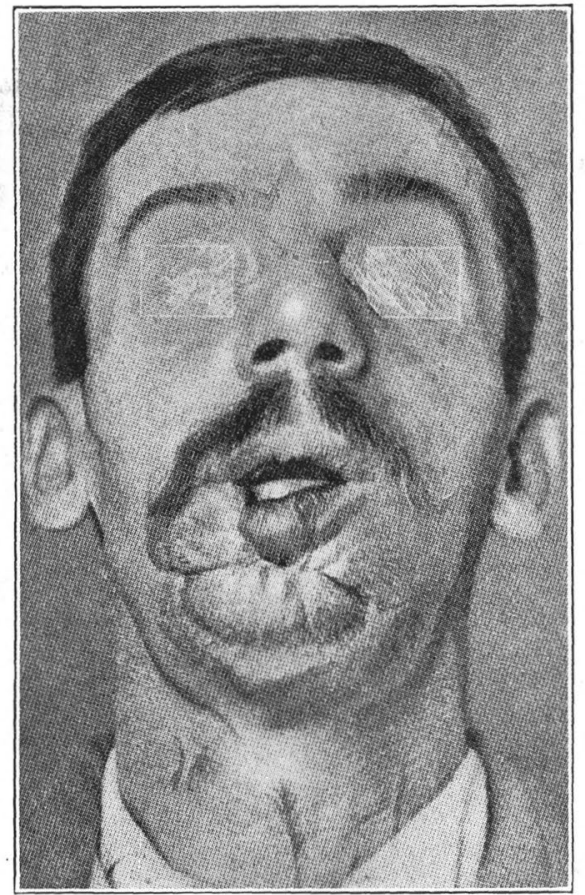

Figure 7 .

may be undetected in the soft parts, time only will reveal these sources of infection, but the preliminary surgical toilet of the mouth will only be slightly impaired by such an oversight and the general principle holds good. This preliminary treatment should be followed up by constant irrigation.

It seems unwise to lay down any hard and fast rules as to extractions near the site of fracture. One must necessarily be guided by circumstances. Even a septic tooth may have to be retained to from a point d'appui to rotate, evert, or invert a fragment which otherwise would have to be left to take its abnormal, possibly, unfavorable, position of convenience. Remove such teeth at the earliest opportunity by all means, but do not ruin a result by too early radical treatment. 'The second step to all possible cleanliness is a correct assembly of all

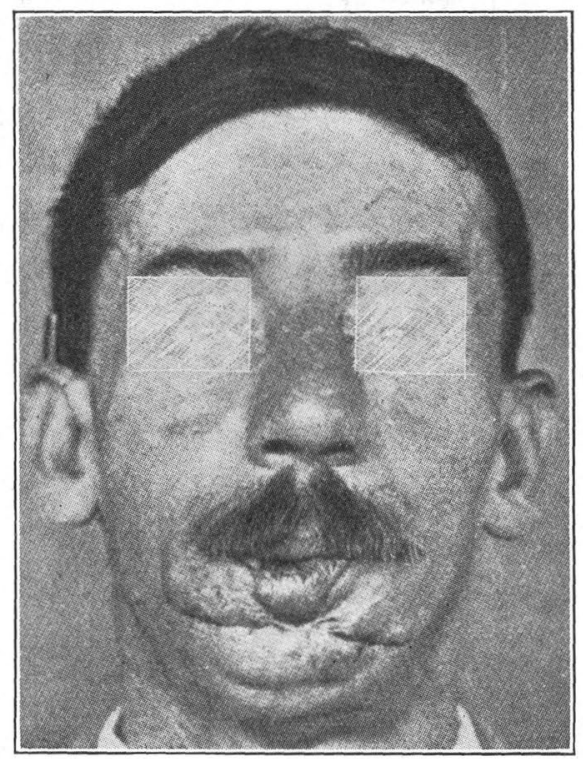

Figure $7 \mathrm{~A}$

misplaced fragments, and experience again teaches that the nearer the fragments can be placed to normal contact, the quicker good results are obtained. It is a curious thing about jaw work that, whenever one tries to lay down a fundamental principle, it always seems to be upset by the next half-dozen cases one sees. A Canadian, a returned prisoner of war, was seen who had been successfully treated for a fracture of the mandible at the angle, still retaining a septic root which had actually given rise to an abscess and had not been removed. 
With indifferent dentures such as one so frequently sees in an English soldier, where grave comminution is present, accompanied by profuse suppuration and possible loss of substance, contact, even at the loss of ideal occlusion, produces quicker and more satisfactory results than ideal occlusion and the doubtful

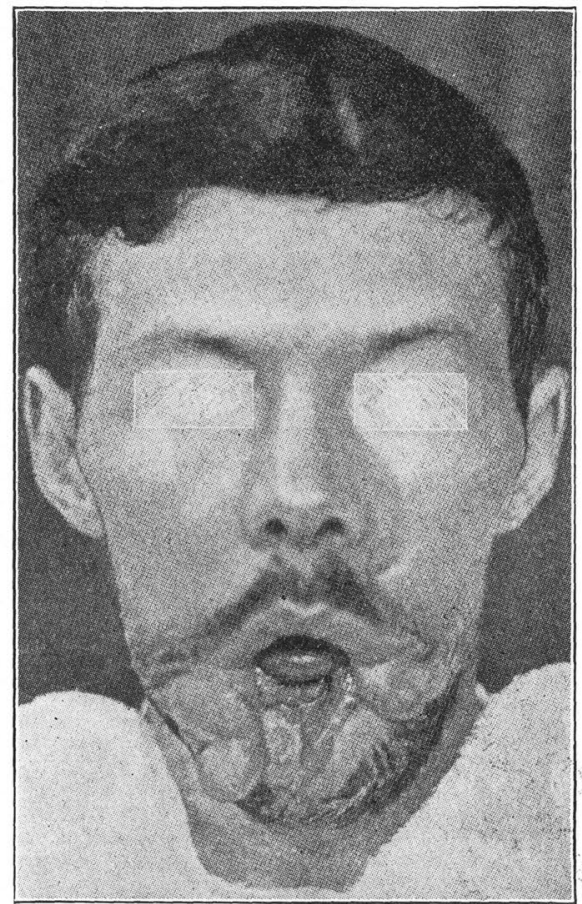

Figure 8.

utility of bone-grafts. One has yet to see the successful bone-graft where an ideal occlusion has been obtained. We feel, therefore, that it is largely a matter of degree of asymmetry or mal-occlusion, and the personal equation comes very largely into play. Touching the subject of comminution, I heartily endorse all that has ever been said since the first days of the war as to the danger of surgeons being too drastic in their treatment. It is a very grave responsibility for a surgeon to remove any pieces of bone that are attached by periosteum, however loose they may appear to be. One is confident that many such pieces form a bridge upon which new bone is built, or centers from which osteogenesis radiates. It is a regrettable fact that satisfactory treatment for the posterior edentulous fragment is still lacking; external transfixion of the ascending ramus has never

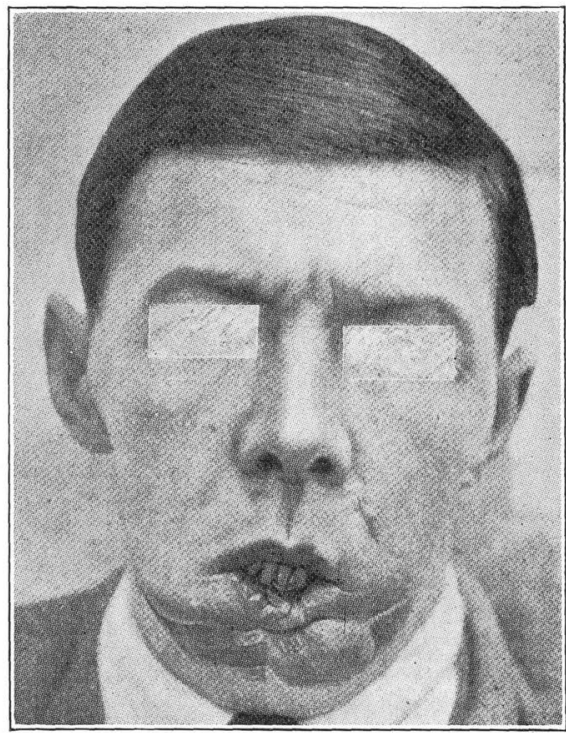

Figure 8 A.

been attempted with us, and all the cases with much loss of substance suitably treated by bone-graft have been cases of this type. In these cases, even where union is complete, much shortening frequently occurs from the upward and forward movement of the uncontrolled fragment and renders the management of a bolus of food on the injured side a practical impossibility when artificial teeth are inserted. The ideal union as demonstrated by sawing thru a morbid specimen is seldom seen in gunshot 


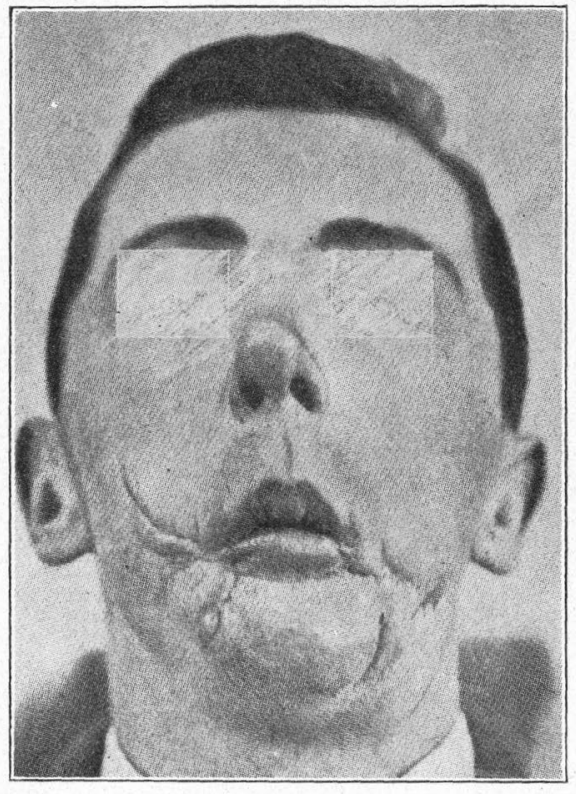

Figure 9.

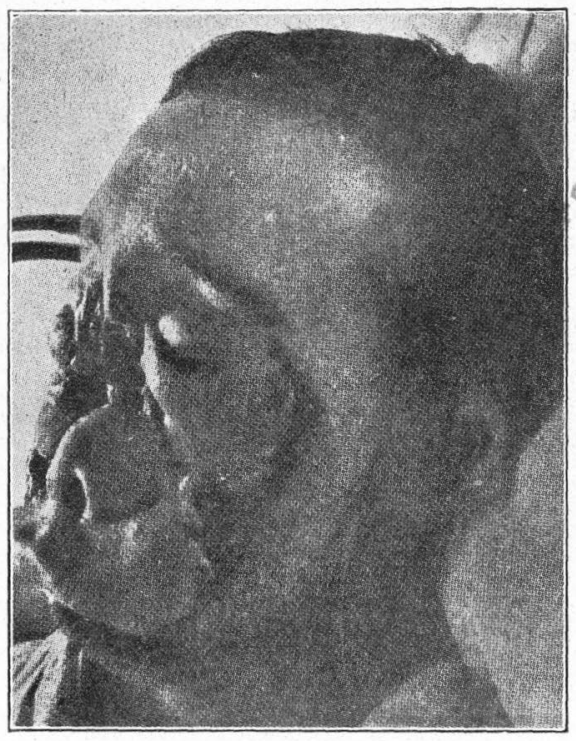

Figure 10.

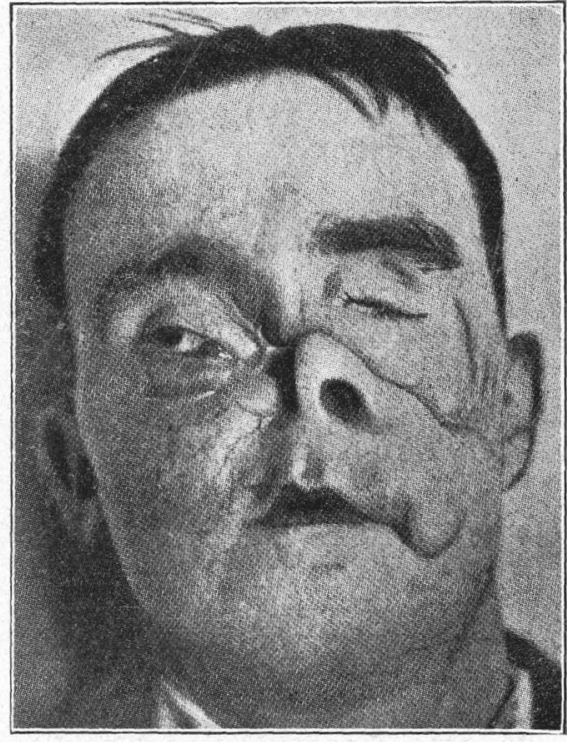

Figure $10 \mathrm{~A}$.

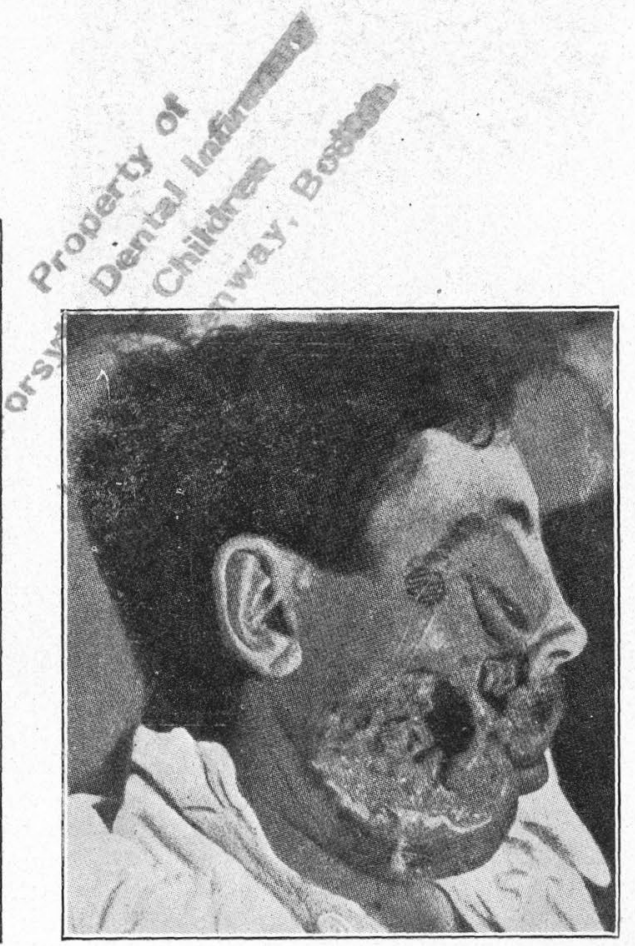

Figure 11. 
wounds; only too often, contact is missed altogether.

Another point that experience has taught is the removal of cheek teeth in order to let the lips approximate when there has been much loss either of bone or soft parts in the region of the symphysis (Figures 7 and 7A). Frequently in restoring the lower lip line the plastic

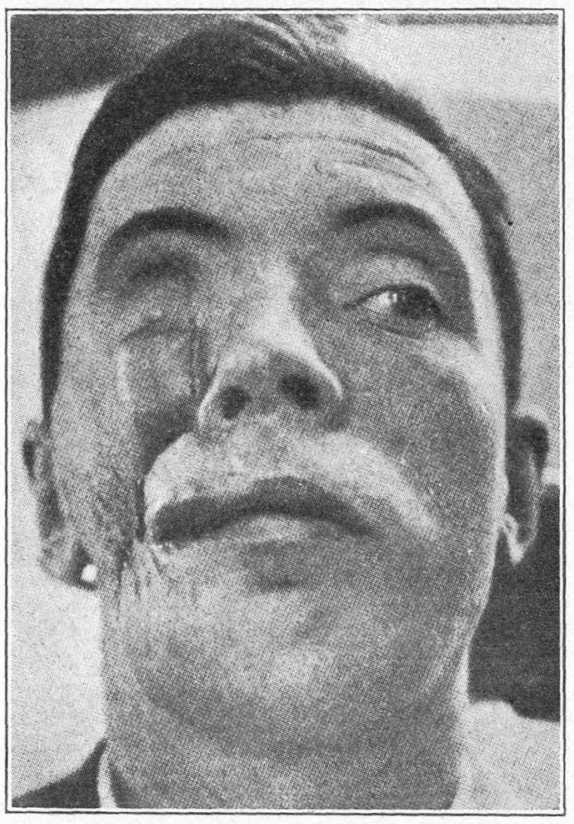

Figure 11 A.

operation involved lowers the lip line so much that this is the only method that can be adopted to bring the lips into contact (Figures, 8, 8A, and 9.) We would appeal to all plastic surgeons in consequence of this, and many similar operations, to make flaps big enough to avoid, as far as possible, this contraction of the buccal orifice. This result is shown in photographs and casts.

In fractures of the mandible only, cemented cap splints have been largely used, the displaced fragments first having been reduced as nearly as possible to normal. The attelle has not been used as much because of its greater tendency to become uncemented. Recourse has been had to immobilization only where the posterior fragment or fragments are edentulous. The method of reduction depends very largely on the condition of the individual patient, the time elapsing between the date of injury and the commencement of the treatment and the mobility of the fragments; and varies from the simple ligature of wire or grass line, the reconstruction of a mode! to normal and the forcing of the fragments into a splint so construcied, to the more elaborate methods founded on the principles developed in ordinary orthodontic practice. Simplification and cleanliness of apparatus are always the first aim.

In treatment of fractured maxilla, time is an even more important factor, the reduction of the underlying bone being more difficult, while the soft tissues cicatrize very rapidly, often causing irreducible malformations of the palate (Figures $10,10 \mathrm{~A}$ ). The antrum is very often involved, and the necessity of an intranasal operation is indicated where chronic suppuration of this sinus persists. The closure of small openings by operation is of doubtful utility, as no cases have yet been seen where a denture was not required, owing to the loss of teeth, before or at the time of injury, and this effectually closes any opening in the hard palate. In cases of greater loss surgical interference is out of the question, and one must resort to the usual restorations as practiced for excision of the maxilla for malignant disease. No case of loss of the soft palate has yet occurred at this center. A graduated series of vulcanite plates seems to fulfill the requirements of most cases, often accompanied by the use of chin and skull caps. Where the whole maxilla has been wrenched off its bony base, the Hamilton bandage and the upper Hayward splint have been used to advantage. This allows mobility of the lower jaw, and the calvarium is used as a point d'appui.

In fractures of both mandible and 
maxilla some form of double Gunning splint must be adopted, being necessarily removable. If there is no displacement in the upper jaw and the teeth only require to be steadied, the splint may consist of upper and lower metal caps soldered together. If there is gross dis- and head net, which has been the method most favored for immobilization at this center. A broad rubber elastic bandage has also been used, and a Hamilton bandage with chin piece.

One result has been seen to-night which can be obtained without bone-

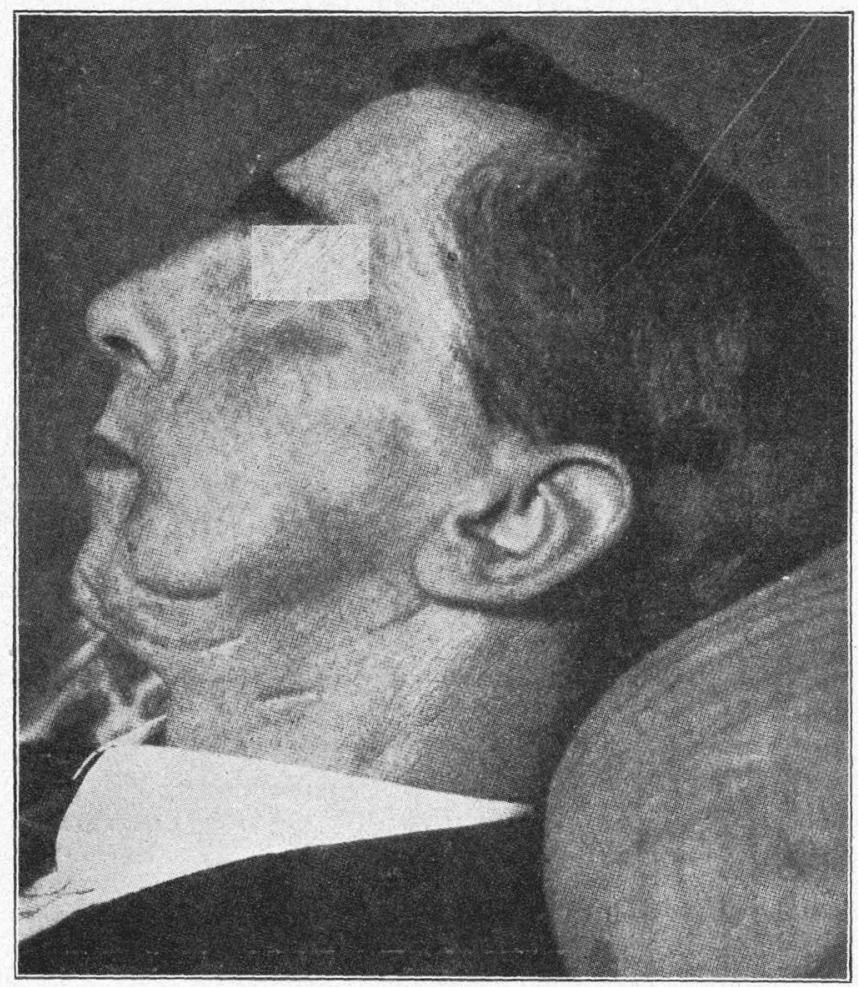

Figure 12 .

placement of the palate, or perforation, a vulcanite upper with a metal lower cap can be used with advantage. If the fracture is accompanied by extensive laceration of the soft parts an interdental extension has been found of great service in preventing subsequent closure from cicatricial contraction, and as an aid to feeding where the occlusion is nearly perfect (Figures 11 and $11 \mathrm{~A}$ ). This should be accompanied by some form of chin support, such as the aluminum chin cap grafting (Figure 9). This result has come about in just one year. Another case has been shown of a patient wounded in August, 1914, who was operated on by Lindemann and Bruhn at Düsseldorf in March, 1915, and supplied with a tibial graft under local anesthesia, the graft being fixed by ivory pegs. In this case the posterior fragment was edentulous and no external fixation was used. As far as one can gather union was not satisfactory at the medial end, for the 
patient having been interned in Switzerland on December 14, 1916, was operated on in February, 1917, for the excision of bone and hair from the anterior end of the graft. In May, 1917, Profes- new dentures will prove of greater service.

\section{Discussion.}

Major W. McAdam Eccles: I would emphasize two points. It is preferable

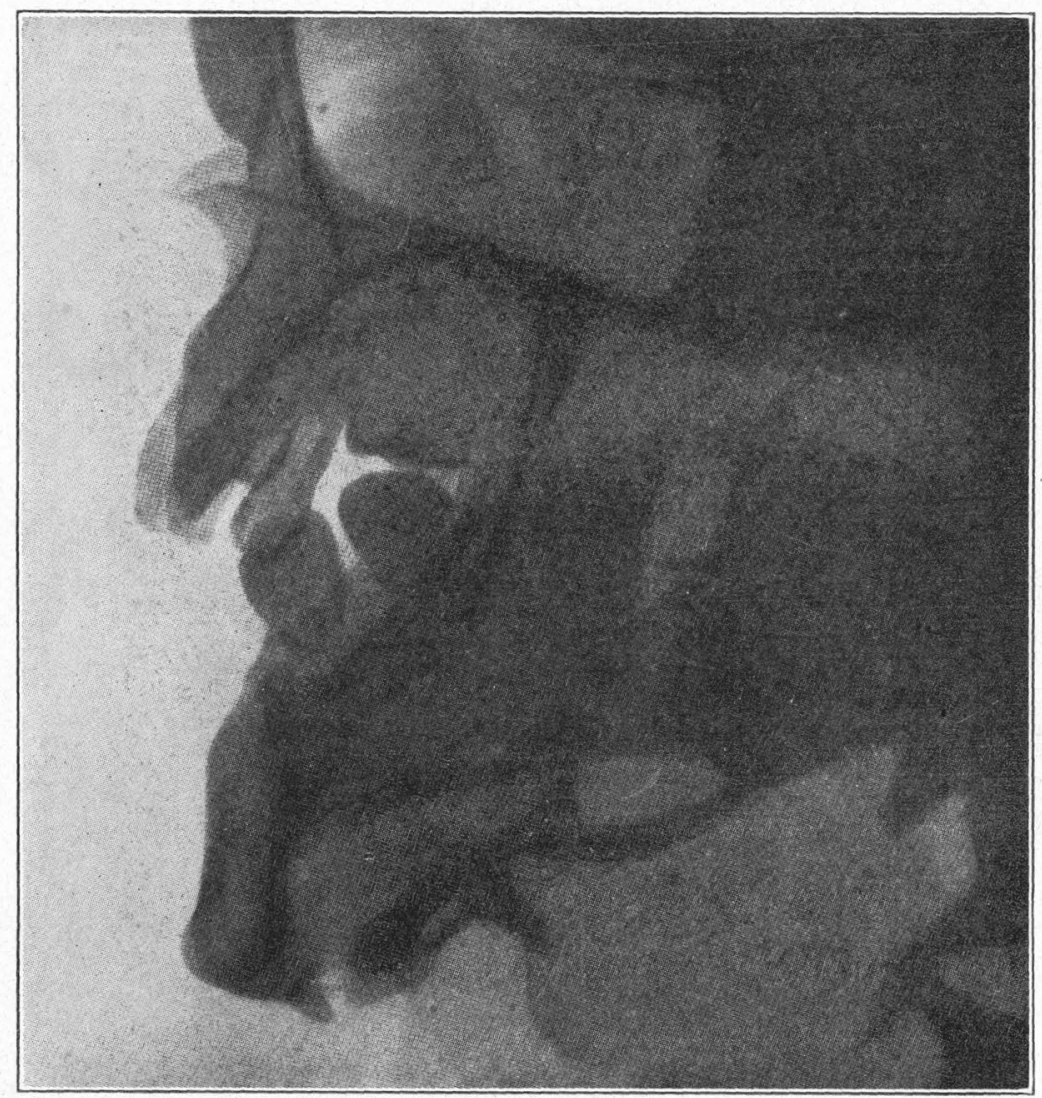

Figure 13.

sor Matti inserted a graft from the crest of the ilium, the skiagram of which can be seen (Figures 12 and 13). Four dentures have been constructed unsuccessfully already, and the man at present has only two right lower molars to chew with. Presumably, the failures were brought about by failing to recc ize that the left cheek was paralyzed, anr that a bolus of food could not be deni: with on that side. It is hoped that the that a "Jaw Department" should be attached to a general military hospital, for then cooperation can be most fully obtained. It is highly advantageous to the patient for the surgeon not to be in a hurry, not to perform plastic operations too early, and not to hurry over their performance when the proper time has arrived for operation. Some of the resilts brought about largely by Nature herself are truly wonderful, and she can 
be helped towards the end frequently a great deal better than at the beginning.

Mr. William Hern: Mr. Northcroft is right in laying down as a first consideration in the treatment of fractured jaws the removal of septic foci, such as retained and decaying roots, but he has not referred to the importance of keeping the gums healthy by frequent and regilar friction with the tooth-brush. The enforced absence, or limitation, of movement of the jaws in these cases, combined with the soft diet necessitated, abolish all natural friction, so that the gums soon become soft, spongy, and infective. The prevention of these conditions is a very important part of the treatment, and of real value for speedy healing. In regard to the treatment of fractures of the mandible in which the posterior fragment is destitute of teeth, to which Mr. Northcroft has referred, the best plan is to wire the fragments together by an operation which does not involve a communication with the mouth. Cases treated at No. 3 General Hospital, Wandsworth, in this manner by my surgical colleagues under my advice have resulted in good bony union.

Mr. A. H. Parrott: There is a variety of splint which I have found effective in many cases of mandibular fracture, single or multiple. Cap splints are fitted to the lower fragments and upper teeth. To these are soldered hooks for traction, if needed, with rubber bands, or wire; when brought to correct occlusion the splints are locked together by a straight wire on either side inserted into tubes previously soldered to both upper and lower splints in corrected position, and divided into sections (like an ordinary hinge) at points most convenient. Hooks are advisable in case of excessive strain on the tubes. The method often obviates unnecessary removal of splints for alteration.

Mr. Doubleday: Does Mr. Northcroft put up his fractures in an open or in a closed bite position; and is he content with a small splint over a few teeth, or does he use a larger one? Does he make any use of the newer lotions such as eusol, or is he content to have his cases treated by older methods, such, for example, as normal saline followed by peroxide of hydrogen?

Mr.G.Northcroft (in reply): Mr. Hern referred to a point certainly omitted from the paper, but the importance of healthy gums is constantly impressed on patients when instructing them in the care of their own mouths. Mr. Parrott's tube and pin method is good, but wire ligatures attached to upper and lower hooks when opposite often seem as satisfactory. The use of open or closed bite depends on the case, the latter for choice. When cap splints are used large ones are found more generally useful where the back teeth can be correctly modelled. A variety of fluids have been used for irrigation, and when extreme foetor is present some deodorant is indicated, but the general practice is to syringe with warm normal saline, very frequent irrigation being the essential point.--Proceedings of the Royal Society of Medicine. 